\title{
Changes in Pain from a Repetitive Thermal Stimulus: The Roles of Adaptation and Sensitization
}

\author{
Mark Hollins ${ }^{a, b},{ }^{,}$, Daniel Harpera, and William Maixner ${ }^{b, c}$ \\ a Department of Psychology, University of North Carolina at Chapel Hill, Chapel Hill, NC 27599 \\ b Department of Endodontics, School of Dentistry, University of North Carolina at Chapel Hill, \\ Chapel Hill, NC 27599 \\ ${ }^{c}$ Center for Neurosensory Disorders, University of North Carolina at Chapel Hill, Chapel Hill, NC \\ 27599
}

\begin{abstract}
This study examined processes that contribute to the changing painfulness of a repeatedly presented thermal (heat) stimulus. The 3-s pulses were presented to the side of the hand at a rate of $4 / \mathrm{min}$, too slow to engage wind-up. Over the course of 32 trials, pain intensity (measured by verbal report on a 0-100 scale) first declined and then (in most cases) rose again, indicating adaptation and sensitization, respectively. The magnitude of adaptation grew across a series of three runs, indicating that adaptation has a slow as well as a fast component. The rate of sensitization depended on stimulus temperature, but not on subjective pain intensity; this result implies that sensitization takes place at an early processing stage. Adaptation and sensitization were comparable in participants with fibromyalgia (FM), temporomandibular disorders (TMD), and in healthy controls (HC), indicating these processes occur before the perceptual amplification that characterizes FM and TMD. The ability of vibration to reduce pain has previously been shown to involve segmental inhibition; the finding in the present study that vibratory gating of pain is significantly (inversely) related to the rate of sensitization suggests that the latter also reflects segmental processes. Several lines of evidence thus point to the conclusion that adaptation and sensitization occur at early stages of sensory information processing.
\end{abstract}

\section{Introduction}

When a series of pulses of heat is presented, their painfulness does not remain constant. The changes in pain intensity that occur puzzled researchers [3,6], until it was realized [19] that pain at any point in time is the result of multiple dynamic influences, some of which act in opposition to one another. Price and colleagues [25] demonstrated two such processes, using a rapid series of thermal pulses: First (sharp) pain declined over the course of the series, while at the same time second (aching) pain built up. The drop in first pain was linked to adaptation of A $\delta$ nociceptive afferents, while the increase in second pain — which dominated

\footnotetext{
(C) 2011 International Association for the Study of Pain. Published by Elsevier B.V. All rights reserved.

*Corresponding author. Tel.: +1 919962 2441; fax: +1 919962 2537, mhollins@email.unc.edu.

The authors have no conflicts of interest.

A preliminary report on portions of this study was presented at the May 2009 meeting of the American Pain Society in San Diego, CA.

Publisher's Disclaimer: This is a PDF file of an unedited manuscript that has been accepted for publication. As a service to our customers we are providing this early version of the manuscript. The manuscript will undergo copyediting, typesetting, and review of the resulting proof before it is published in its final citable form. Please note that during the production process errors may be discovered which could affect the content, and all legal disclaimers that apply to the journal pertain.
} 
the overall sensory experience-was associated with the transient increase [20] in the response of dorsal horn neurons to $\mathrm{C}$-fiber input. The latter change, called temporal summation, requires stimulus repetition at inter-stimulus intervals (ISIs) of $3 \mathrm{~s}$ or less [24].

This relatively simple picture of changes in experimental pain from a repetitive thermal stimulus was made more complex by recent findings that adaptation has both peripheral and central components [7], and that pain intensity follows a triphasic time course during the first minute of continuous heat stimulation [12,21], perhaps reflecting a gradual sensitization in addition to temporal summation and adaptation.

The purpose of the present study is to further describe and characterize the processes of adaptation and sensitization. We isolated these processes from temporal summation by spacing heat pulses so widely that the latter phenomenon did not occur.

Pain signals are repeatedly modified as they travel from receptors to the brain. For example, cortical responses to noxious stimulation more strongly reflect individual differences in pain sensitivity than do responses at earlier levels of the neuraxis [2]. We use the term "early" to describe processing stages that precede the incorporation of individual-difference or cognitive information into the pain signal, and refer to stages that follow this transition, such as the perceptual amplification that is associated with hypervigilance [8], as "late". The terms are used here in a functional sense and do not refer to specific anatomical loci.

By determining whether adaptation and sensitization were related to stimulus temperature, clinical status, hypervigilance, and other factors, we sought evidence as to whether these events occur early or late in processing. Patients with fibromyalgia and temporomandibular disorders were included to ensure large individual differences in pain modulation within the sample.

We also examined the ability of vibration to reduce pain, a modest effect $[9,14,23,35,37]$ that primarily reflects segmental inhibition [29,34]. By examining the relationship between this vibratory analgesia and sensitization, we sought additional evidence concerning the stage at which the latter occurs. A significant statistical association between these processes might indicate that sensitization also depends, at least in part, on segmental events.

The ability to empirically distinguish perceptual phenomena that are due to early and late stages of processing is the first step in the development of a psychophysical taxonomy of pain dynamics, that may be useful in characterizing patients whose pain perception is subtly disturbed.

\section{Methods}

\subsection{Participants}

There were 75 participants: 17 with fibromyalgia (FM), 29 with temporomandibular disorders (TMD), and 29 pain-free healthy controls (HC). All were female. Average ages of the three groups were similar: FMs, 37.6 years ( $\mathrm{SD}=12.2$ ); TMDs, 36.7 (11.7); HCs, 37.2 (12.0). A one-way ANOVA showed that these values were not significantly different, $\mathrm{F}(2,72)=.035, \mathrm{p}=.97$. One additional $\mathrm{HC}$ participant was enrolled, but was excluded from data analysis because during the experiments she consistently gave a zero pain-intensity rating to the highest temperature presented $\left(51^{\circ} \mathrm{C}\right)$.

The experiments reported here were part of a larger study of pain responsiveness in these three groups, that included an investigation of hypervigilance [8]. Most subjects participated in three unrelated 2-hr sessions, of which the second was devoted to the experiments reported here, whereas hypervigilance was measured (by means of the Pennebaker Inventory 
of Limbic Languidness [22]) in the third session. Sessions were always on separate days, and usually separated by about a week. Two subjects included in this study dropped out before completing the hypervigilance session. Additional subjects have been run since the publication of the hypervigilance study, accounting for the larger sample sizes in the current investigation.

Procedures for recruiting and screening participants are fully described in [8]. Briefly, participants were recruited with flyers, newspaper advertisements, and (for some with FM and TMD) by clinical referral. Individuals who passed a telephone screening were invited to come to the UNC School of Dentistry for an assessment visit to determine their clinical status; this included self-report measures of pain complaint and medical history, and a physical examination conducted by calibrated and validated clinical examiners. TMD participants met the Research Diagnostic Criteria for TMD [4], and reported orofacial pain of at least one month's duration. FM subjects reported persistent widespread body pain of more than 3 months duration, and pain on palpation at 11 or more of the 18 body sites (tender points) specified by the American College of Rheumatology [36]. Three individuals fell short of this criterion (with 7, 8, and 9 tender points, respectively) but presented with other strong indicators of FM (see [8]). HC participants did not report any significant pain, and did not meet criteria for FM or TMD.

The different levels of everyday pain experienced by members of the three groups are shown by their mean VAS scores on the Short Form of the MPQ [18]: 31.4 (SD=21.0), 11.8 (13.4), and 3.1 (9.7) for FM, TMD, and HC participants, respectively. FM and TMD participants reported being in pain $77.3 \%$ and $42.8 \%$ of their waking day, respectively, while HCs indicated having pain during only $3.3 \%$ of their waking day.

Exclusion criteria for all potential participants were the presence of uncontrolled hypertension; heart, lung, or kidney disease; diabetes; hyperthyroidism; epilepsy; drug or alcohol abuse; or major psychiatric disorder. Individuals were also excluded if they were pregnant or nursing, were taking opioids, or had a condition that would have made it difficult to carry out the experimental procedures. Finally, if any potential participants had had a pacemaker or other cardiac implant device (none did), they would have been excluded because of the possibility of interference from the magnetic fields generated by the minishaker (see below).

Because many of the participants were in intense chronic pain, they were not asked to discontinue their pain medication. Since those with the most severe pain would have been least able to comply with such a request, we judged that it would have led to a sampling bias favoring those with milder conditions. In addition we felt that the stability of an uninterrupted medication regimen was preferable to a situation in which patients might have been intermittently resorting to medication.

Subjects gave written informed consent and were paid for their participation. All aspects of the study were approved by the University's IRB.

\subsection{Apparatus}

Two types of stimulation were employed: Noxious heat, and innocuous vibration.

Vibrations were delivered by a computer-controlled instrument called the Vibrotactile Laboratory System (VLS) that is capable of delivering both vibrotactile and thermal stimuli to the hand of an experimental subject. This custom-built device (Dancer Design, 123 Boundary Road, St. Helens, Merseyside, WA10 2LU, England) uses a minishaker to deliver vibrations through a hole in a small table on which the subject rests her hand. In the present 
study, vibration was presented to the thenar eminence through a flat, round aluminum contactor with a diameter of $8 \mathrm{~mm}$. The contactor was surrounded by a large aluminum plate (outer diameter $64 \mathrm{~mm}$ ) that was embedded in the plastic tabletop. Contactor and surround were separated by a gap of $1 \mathrm{~mm}$. Vibration frequency was either 33 or $100 \mathrm{~Hz}$, values chosen for their ability to preferentially stimulate (although not, at suprathreshold levels, to isolate) the Meissner and Pacinian mechanoreceptive channels, respectively [5]. Water from a remote heating/refrigeration unit circulated through tunnels inside both the contactor and the surround, maintaining them at $32^{\circ} \mathrm{C}$ (approximately resting skin temperature). All aspects of stimulation were under computer control: Using a virtual interface that runs on LabView, an experimenter set the parameters of stimulation and monitored them throughout each experimental run.

The noxious thermal stimuli were presented with a Medoc TSA II NeuroSensory Analyzer. The $16 \times 16 \mathrm{~mm}$ contactor of this computer-controlled device is heated by a Peltier thermode at a rate of approximately $5^{\circ} \mathrm{C} / \mathrm{s}$, and actively cooled by circulating water.

\subsection{Procedure}

2.3.1. Parameter determination-In preliminary measurements, the temperatures and vibration amplitudes to be used in the main experiment were determined.

We first sought to determine a pair of temperatures, differing by $0.5^{\circ} \mathrm{C}$, that would yield verbal pain ratings in, or as close as possible to, the 30-40 range on a $0-100$ scale, where 0 was defined as "no pain" and 100 as "the most intense pain imaginable." A pair of temperatures, rather than a single temperature, was selected, so that stimulation could toggle pseudo-randomly between them during the main experiment; this was done to demonstrate to the subject that stimulus temperature was not always the same. The orders were pseudorandom in that neither temperature was ever presented on more than two trials in a row, and the two temperatures were presented about equally often in the early, middle, and late portions of a run. Each subject participated in three runs (see below), using three different pseudo-random orders, A, B, and C. In order to determine the optimal temperatures for each participant, the experimenter began by presenting a $3-\mathrm{s}, 46^{\circ} \mathrm{C}$ pulse, and then adjusting the temperature of subsequent pulses upward or downward, depending on the subject's response, until the desired criterion was approximated. Temperature returned to a baseline value of $38^{\circ} \mathrm{C}$ between trials.

Two practical considerations limited the precision of these measurements. First, to reduce the number (and therefore the adaptation produced by) these preliminary pulses, adjustments smaller than $0.5^{\circ} \mathrm{C}$ were not made; second, temperatures above $51^{\circ} \mathrm{C}$ were not used, in order to minimize erythema and other effects on the skin that might, in the main experiment, carry over from one run to the next. If none of the stimuli elicited a rating of 30 or above, a temperature of $51^{\circ} \mathrm{C}$ was used. Perhaps because of these limitations, the mean temperature selected was only slightly lower in $\mathrm{FMs}\left(47.6^{\circ} \mathrm{C} ; \mathrm{SD}=2.0\right)$ than in $\mathrm{HCs}\left(48.9^{\circ} \mathrm{C} ; 2.3\right)$, with that for TMDs $\left(48.5^{\circ} \mathrm{C} ; 2.6\right)$ being intermediate; the difference among these values was not significant, $\mathrm{F}(2,72)=1.51, \mathrm{p}=.23$. The overall average temperature was $48.5^{\circ} \mathrm{C}(\mathrm{SD}=2.4)$, and the temperatures used ranged from 41 to $51^{\circ} \mathrm{C}$.

In the case of vibration, amplitudes of $40 \mathrm{~dB}$ SL (i.e., $40 \mathrm{~dB}$ above threshold) were to be used in the main experiment. To determine these amplitudes, vibrotactile thresholds at $33 \mathrm{~Hz}$ and $100 \mathrm{~Hz}$ were measured, in the absence of noxious thermal stimulation. The contactor was positioned for vibratory stimulation (here and in the main experiment), by being slowly raised until an uptick in the force readout showed that contact had been made with the thenar of the subject's right hand, which rested palm down over the opening in the VLS tabletop. The contactor was then raised an additional $1 \mathrm{~mm}$, under computer control, to ensure firm 
contact with the skin during vibration. Vibrotactile thresholds were measured with 2-interval forced-choice tracking, using parameters of stimulation (each trial consisted of a 1-s pulse of vibration randomly presented in either the first or the second of two visually-indicated intervals) and a tracking algorithm (step up following an error; step down following three correct responses, not necessarily consecutive) closely comparable to those employed in our earlier research $[9,10,11]$. Runs were 40 trials in length, and threshold was defined as the mean log amplitude during trials 33-40.

2.3.2. Main experiment $-A$ run in the main experiment consisted of a series of thermal pulses, presented by means of the Medoc stimulator to the side of the base of the thumb, in a region of skin that was transitional between hairy and glabrous. Pulses were $3 \mathrm{~s}$ in duration, and were separated by intervals of $12 \mathrm{~s}$, so that they occurred at the rate of $4 / \mathrm{min}$. The thermal contactor was held stationary against the skin, and between pulses its temperature was at a baseline value of $38^{\circ} \mathrm{C}$. When a pulse began, temperature rose, at a rate of approximately $5^{\circ} \mathrm{C} / \mathrm{s}$, to the desired temperature, where it remained for the rest of the 3-s pulse duration, thereafter declining (by active cooling) back to baseline.

Most runs consisted of 33 trials (Fig. 1), with the thermode applied to the same location throughout. All but one of the trials employed one of the two temperatures (differing by $0.5^{\circ} \mathrm{C}$ ) selected on the basis of preliminary pain ratings. The odd trial (trial 3) was a probe (administered to all but the first seven participants), on which a temperature $2^{\circ} \mathrm{C}$ below the lower of the two standard temperatures for that subject was presented. This probe trial served two functions. First, it allowed us to confirm that the subjects' responses were under good stimulus control, which was evidenced by the following findings: 1) Paired-samples ttests revealed that pain on the probe trial (mean of all runs containing a probe trial $=20.9$, $\mathrm{SD}=11.9)$ was significantly lower than pain on the immediately preceding $(36.4,15.2)$ and following $(28.2,12.9)$ trials $[\mathrm{t}(67)=11.6, \mathrm{p}<.001$ and $\mathrm{t}(67)=8.6, \mathrm{p}<.001$, respectively]; and 2$)$ In the majority of runs in each group (FM, 78\%; TMD, 74\%; HC, 64\%), probe trial pain was lower than pain on trials just before and after. Second, this trial was used to make clear to the subject that different trials could be expected to involve different temperatures. This probe trial was omitted from the figures (except Fig. 1), and from further statistical analysis of the data, as were a small number of extra trials in some early subjects.

A run, omitting the probe trial, thus consisted of 32 trials, and can be described as consisting of an early phase (trials 1-12), a middle phase (trials 13-22), and a late phase (trials 23-32). In some runs, continuous vibration was presented during the middle phase: It began as soon as the subject gave her response to trial 12 , and continued until she gave her response to trial 22. Subjects were told to rate (on the 0-100 scale) the intensity of pain produced on each trial by the thermal stimulation, and to ignore any vibration that was present.

Each of the 75 subjects participated in three runs, separated by 10-min breaks. In one of these, $33 \mathrm{~Hz}$ vibration at $40 \mathrm{~dB}$ SL was used; in another, $100 \mathrm{~Hz}$ vibration at the same sensation level; and in another, no vibration was presented. The order of the three runs was randomly assigned, with the constraint that within each participant group, the six possible orders were (to the extent possible) used an equal number of times. The pseudo-random order of two temperatures within a run was also counterbalanced so that orders A, B, and C were used about equally often for each run type.

\section{Results}

\subsection{Time Course of Experimental Pain Ratings}

Participants showed systematic changes in their pain ratings over the course of a run (Fig.

2). In most cases ( $74 \%$ of all runs), pain ratings declined during the first few trials, 
indicating adaptation to the thermal stimulus. After this initial phase, there was considerable diversity among individuals in the trend(s) shown in later trials; on average, subjects reported a gradual increase in pain. The trials are too widely spaced for this increase to be the result of wind-up, and we therefore refer to it as sensitization.

We consider first the adaptation phase, then the sensitization phase. Finally, we examine the effect of vibration on pain intensity.

\subsection{Short-term and Long-term Adaptation}

To quantify the adaptation that occurred at the beginning of a run, we made use of the fact that it appeared to be a negative exponential process, with pain intensity dropping rapidly at first and then more slowly (see Fig. 2). If the function asymptotically approached zero, and its parameters remained constant across individuals, then pain intensity would always drop by the same fraction in the first (e.g.) 2 minutes of the run, regardless of the level at which it began. Neither of these conditions was met: In particular, pain for most subjects approached an asymptote substantially greater than zero. Nevertheless, the absolute decline in pain during adaptation (i.e. the mean of ratings 7 and 8 subtracted from the mean of the first two ratings) was closely related to the initial level of pain [r=.63, $\mathrm{p}<.001]$, implying that the use of relative decline (absolute decline divided by the mean of the first two ratings) as a measure of short-term adaptation would factor out much of the between-subject variance attributable to differences in initial pain ratings, and thus provide a more stable measure.

In addition to this short-term adaptation that occurred within a run, a longer-term adaptation manifested itself when a participant's three runs were examined together (Fig. 3). Pain intensity, averaged over all trials within a run, declined markedly as the session proceeded. A $3 \times 3$ mixed model ANOVA examining the effects of run and clinical group showed that average pain intensity dropped significantly $[\mathrm{F}(2,144)=7.12, \mathrm{p}=.001]$, but that this drop did not differ across groups (interaction: $\mathrm{F}(4,144)=.61, \mathrm{p}=.66)$. The main effect of clinical group on pain intensity was also not significant $[\mathrm{F}(2,72)=.002, \mathrm{p}=.998]$, as is to be expected from the fact that temperature was adjusted for individual subjects to produce comparable levels of initial pain.

The drop in average pain ratings from one run to the next occurred despite the fact that the initial pain ratings (average of trials 1 and 2 ) for each run did not change significantly between Runs 1 (35.0; $\mathrm{SD}=18.0), 2(36.0 ; 18.0)$ and 3 (38; 18.3), $\mathrm{F}(2,144)=1.90, \mathrm{p}=.15$. The overall decline in pain across runs was the result of a gradual increase in the magnitude of short-term adaptation: Pain decreased an average of $16 \%$ during the first 8 trials of Run 1, as compared with $25 \%$ in Run 2 and $38 \%$ in Run 3 . This pattern was present in the data of all three groups of participants. A $3 \times 3$ mixed model ANOVA, with the relative drop in pain during the first 8 trials of a run as the dependent variable, and run and group as the factors, showed that the effect of run was highly significant $[\mathrm{F}(2,144)=10.1, \mathrm{p}<.001]$, but neither the effect of group $[\mathrm{F}(2,72)=.75, \mathrm{p}=.47]$ nor the interaction of run and group $[\mathrm{F}(4,144)=.23$, $\mathrm{p}=$. 92] was significant. Thus both the fast component of adaptation (the decline in pain within the first few trials of a run), and its slow component (the fact that the fast component increased from one run to the next) were comparable in FM, TMD, and HC participants.

\subsection{Sensitization}

To quantify sensitization, we fit a regression equation to the ratings for trials $10-32$ for each subject (resembling the regression line fit to the mean data in Fig. 2), and determined its slope, in terms of units on the $0-100$ pain intensity scale per trial.

The temperatures used for each subject were chosen, on the basis of preliminary trials, to elicit ratings within (or as close as possible to) the 30-40 range on the pain intensity scale, 
without exceeding $51^{\circ} \mathrm{C}$. The selected temperatures varied widely (see Fig. 4). Interestingly, there was a significant positive relationship between a participant's rate of sensitization and the temperature used in her runs $(r=.48, p<.001)$.

In a given subject, pain intensity generally increases with temperature, and the data in Fig. 4 might therefore be taken to suggest that sensitization magnitude depended on the intensity of pain being experienced at the beginning of the summation process. To test this possibility, we compared sensitization slope with pain intensity at the start of sensitization; no relationship was found between these variables, $r=-.05, p=.70$ (see Fig. 5). The fact that the magnitude of sensitization was related to stimulus temperature, but not to the level of pain that temperature evoked, suggests that the underlying mechanism is an early one, prior to the locus (or series of loci) in the nociceptive pathway at which pain signals come to reflect subjective intensity.

Given the association reported in some earlier studies between the speed or magnitude of temporal summation and clinical status, we examined the possibility that the rate of sensitization would be generally higher in the TMD and especially the FM patients than in the healthy controls. In fact, this was not the case: The rate of sensitization did not vary significantly as a function of group, $\mathrm{F}(2,72)=1.42$, $\mathrm{p}=.25$. This result is consistent with the view that sensitization and temporal summation are separate processes.

If the sensitization documented in the present study reflects an early stage of pain processing, we would expect it to be impervious to cognitive influences such as hypervigilance, which has been shown —in many of these same subjects [8]— to be a powerful agent of late perceptual amplification. To test this prediction, we compared sensitization scores with scores on the Pennebaker Inventory of Limbic Languidness (PILL) [22], the measure of hypervigilance used in our earlier study. Most of these PILL scores, obtained in a separate session, have been reported previously [8], but the two samples do not exactly correspond, mainly because additional subjects were enrolled after the earlier study was published. ANOVA revealed a highly significant difference in hypervigilance between the clinical groups, $\mathrm{F}(2,70)=30.33$, $\mathrm{p}<.001$, consistent with our earlier report [8].

Nevertheless, PILL scores did not significantly correlate with sensitization, $r=-.09, p=.45$, implying that sensitization occurs below the reach of this cognitive influence.

\subsection{Modulation of Experimental Pain by Vibration}

For a period of 10 trials (trials 13-22) in the middle of some runs, a vibratory stimulus was applied to the thenar eminence. This vibration began immediately after the subject's rating of the thermal stimulus on trial 12, and continued without interruption until she had rated the thermal stimulus on trial 22. Each subject participated in one run with $33 \mathrm{~Hz}$ vibration and another with $100 \mathrm{~Hz}$ vibration, in both cases at an intensity of $40 \mathrm{~dB}$ SL. Evidence of pain modulation consisted of a deviation of pain ratings above or below the trend line formed by trials preceding and following the vibration period.

In many subjects, there was a small deviation (positive or negative) of pain ratings during the vibration period. To determine whether this represented genuine modulation of pain by vibration, or merely trial-to-trial variability, we derived quantitative modulation scores that could be subjected to statistical analysis. A modulation score was obtained for each run by (a) calculating the mean pain rating during the 10-trial vibration period; and (b) subtracting from this the combined mean of the seven trials immediately preceding, and the seven trials immediately following, the vibration period. Mean modulation scores suggested a slight reduction of pain during $33 \mathrm{~Hz}(-1.1 ; \mathrm{SD}=3.7)$ and $100 \mathrm{~Hz}(-0.4 ; 5.4)$ vibration periods, but not during the corresponding 10 -trial period in control runs $(0.5 ; 2.5)$. 
A repeated-measures ANOVA comparing modulation scores from the three types of runs ( $33 \mathrm{~Hz}$ vibration, $100 \mathrm{~Hz}$ vibration, and control) found a significant effect of condition, $\mathrm{F}(2,148)=3.71, \mathrm{p}=.027$. Post-hoc comparisons of modulation scores from vibration runs with those from control runs showed the difference to be significant at $33 \mathrm{~Hz}, \mathrm{t}(74)=3.00, \mathrm{p}=.004$, but not at $100 \mathrm{~Hz}, \mathrm{t}(74)=1.35, \mathrm{p}=.18$. Subsequent analysis was therefore confined to the effect of $33 \mathrm{~Hz}$ vibration, although results at the two frequencies were qualitatively similar. The shaded area in Fig. 6 shows that pain gating was a modest effect that lingered into the post-vibration period.

Pain modulation scores were marginally correlated with temperature [r=.22, $\mathrm{p}=.057]$, meaning that there was a tendency for pain elicited by low temperatures to be more effectively suppressed than pain elicited by higher temperatures. Modulation scores were not, however, related to pain intensity on the trial (\#12) immediately preceding the onset of vibration $[\mathrm{r}=-.15, \mathrm{p}=.21]$ and they did not differ by participant group, $\mathrm{F}(2,72)=.58, \mathrm{p}=.56$. This pattern of statistical outcomes, similar to that obtained for sensitization slope, implies that pain gating is likewise a relatively low-level process.

Finally, there was a small but significant correlation between pain modulation score in the $33 \mathrm{~Hz}$ run, and the slope of sensitization in the same participant's control run [r=.23, p=. 046]. Vibration tended to increase pain in individuals with high sensitization slope, but to decrease pain in subjects with lower values of this continuous variable.

\section{Discussion}

This study has examined the dynamic events that occur when a series of widely spaced noxious thermal stimuli is presented. In this situation pain intensity follows a bi-phasic time course, consisting of adaptation followed by sensitization.

\subsection{Characterizing adaptation and sensitization}

The present study sheds light on the nature of these processes, in four ways.

First, it was found that the process of adaptation has short-term and long-term components. Each subject participated in three runs, that is, three 32-trial series of noxious stimuli, with breaks of 10 min between runs. Within the first (approximately) eight trials of each run, pain declined rapidly, following a negative exponential time course, but recovered to its original level by the beginning of the next run. However, adaptation constituted a greater drop in pain in the second run than in the first, and in the third than in the second. That is, the extent of adaptation increases if the process is repeatedly engaged.

A second set of findings concerns the fact that sensitization of the type studied here, which can be quantified by the slope of the change in pain intensity over the course of postadaptation trials within a control (i.e., no-vibration) run, varies considerably from person to person—an observation made also by Naert et al. [21]. In some individuals, pain increases substantially, in others it holds steady, and in still others it declines.

In the present study, the rate of change in pain intensity-that is, the strength of sensitization -is closely related to stimulus temperature, rather than to initial pain intensity (compare Figs. 4 and 5). This result indicates that sensitization occurs before the nociceptive signal has been modified to reflect pain intensity, rather than stimulus intensity, and that sensitization may therefore be described as an early process. Neuroimaging indicates that, at least under some conditions, the cortical response to a noxious stimulus is closely related to subjective pain intensity [2]; our results therefore suggest that sensitization occurs at a precortical level. 
Third, the magnitude of sensitization is statistically equivalent in FM, TMD, and HC participants; the same is true of both the rapid and slow components of adaptation. These results imply that adaptation and sensitization take place at a stage prior to that at which hypervigilance-related perceptual amplification occurs, because the extent of this amplification, like hypervigilance itself, has been shown to differ across these three clinical groups [8].

Recent evidence indicates that the perceptual amplification accompanying another cognitive process, catastrophizing, occurs in the brain [27]. It is therefore reasonable to infer that the same is true of hypervigilance-induced perceptual amplification. In any case, adaptation and sensitization do not appear to be influenced by these cognitive factors, even via descending influences. This conclusion is consistent with the fact that peripheral changes can contribute to both adaptation [1,7,24,32] and sensitization [15].

Another possible contributor to the sensitization observed in the present study is a release from inhibition of $\mathrm{C}$ fiber-mediated pain responses as each experimental run progressed. Price and colleagues [25] showed that $\mathrm{C}$ fiber-mediated pain is increased following A-fiber blockade by nerve compression. In addition, they found that peripheral A $\delta$ fiber responsiveness is suppressed for up to 80 s following brief stimulation. Taken together, these two discoveries by Price et al. suggest that sensitization in the present study could reflect, at least in part, a progressive increase in $\mathrm{C}$ fiber-mediated pain as A $\delta$ fibers gradually adapt to the heat pulses.

The finding that sensitization is comparable in FM, TMD, and HC participants serves to emphasize the difference between this phenomenon and temporal summation, which has repeatedly been shown to be enhanced in patients with idiopathic pain disorders $[13,17,26,30$; but see 33]. A comparison of temporal summation and sensitization in the same participants will be needed to definitively establish similarities and differences (other than time course) between these two processes.

A fourth way in which the present study adds to knowledge of pain dynamics concerns the ability of vibration to reduce pain. Vibratory analgesia is statistically demonstrable in group data, even though it is not always present in individual records; in fact, we confirm earlier reports $[16,28]$ that vibration sometimes increases rather than decreases pain. The term vibrotactile pain modulation, rather than vibratory analgesia, is therefore suggested for future use. The average pain modulation score in the present study was negative compared to a control run, i.e. vibration reduced pain, but the effect was significant only at $33 \mathrm{~Hz}$ (not $100 \mathrm{~Hz}$ ), perhaps because high-frequency sensitivity varies as a function of age, menstrual cycle, and other observer characteristics [5]. Nevertheless, results for the two vibration frequencies were qualitatively similar.

An additional finding in the present study is that there is a significant correlation between sensitization and pain modulation. That is, the lower the slope of the sensitization function, the greater the tendency for vibration to reduce pain. Given the evidence linking this type of antinociception to segmental inhibition [29,34], its statistical relationship with sensitization suggests that the latter process may, at least in part, occur at the segmental level as well. We cannot, however, rule out the possibility that peripheral sensitization also contributed to the observed effects. In any case, the result is an additional piece of evidence that sensitization occurs at an early stage of nociceptive signal processing.

\subsection{Comparison with earlier studies}

The time-course of changes in pain that we obtained is somewhat simpler than the triphasic response to continuous thermal stimulation described by King et al. [12]. An initial pain 
increase in their study was attributed to "temporal sensitization," although a change in thermode temperature may also have contributed to it. Pain subsequently decreased, presumably reflecting adaptation. Finally, pain rose again, a shift resembling the gradual sensitization that we observed. Their report systematically documents the occurrence of sensitization in both healthy control participants and chronic pain patients. When present, DNIC reduced pain similarly in all phases [12]; in our view, this suggests they occur early enough to be influenced by descending modulation.

By using a series of brief thermal stimuli and presenting them at a slow (4/min) rate, we prevented the occurrence of temporal summation. In its absence, there appears to be a correspondence between the two phases of the time course we recorded, and components 2 and 3 of the response profile obtained with continuous stimulation [12,21]. The fact that all three studies found adaptation followed by a gradual sensitization indicates the robustness and generality of these two processes.

A previous report [7] demonstrated central and peripheral components of adaptation, using separate conditions in which the thermode was either moved about, or kept at the same location, across trials. The peripheral component had a time constant of about $10 \mathrm{~s}$, the time between pulses; the slow component had a time constant roughly twelve times this. The time constants in the present study are considerably slower: Rapid adaptation appears to have a time constant of about $45 \mathrm{~s}$, or three trials (see the exponential function in Fig. 2). The time constant of the slow component in the present study cannot be accurately determined but appears to be at least $10 \mathrm{~min}$. These differences in time course may result from the different pulse shapes and intervals in the two studies.

A more substantial difference between our results and those reported by Greffrath et al. [7] is that sensitization was not in evidence in the earlier study. The most likely explanation for this is that Greffrath et al. used only mild-to-moderate stimuli. Their careful calibrations showed that, although the brief pulses produced with the CHEPS stimulator had nominal peak temperatures as high as $51^{\circ} \mathrm{C}$, actual thermode temperature at the skin did not rise above $45.2^{\circ} \mathrm{C}$. Given our finding that sensitization increases with temperature, it is reasonable to conclude that the adaptation process was isolated in the earlier study by the restricted range of temperatures employed.

\subsection{Conclusion}

Taken in conjunction with earlier work on FM and TMD, including a report from our lab on hypervigilance and perceptual amplification in many of the same individuals [8], the results of the present study suggest that it is possible to psychophysically distinguish between stages of nociceptive information processing that occur relatively early (peripherally or at a segmental level) and others that occur at a higher level. The fact that adaptation, gradual sensitization, and vibratory pain modulation did not differ significantly across the three groups of participants (FM, TMD, and HC) indicates that these processes are relatively undisturbed in TMD and FM. Furthermore, the stimulation procedure used in this study, a series of pulses of noxious heat separated by long ISIs, makes possible a fractionation of low-level processes by selectively activating some of them (adaptation and sensitization) without engaging temporal summation. This protocol may therefore contribute to the systematic examination of pain processing algorithms and their relationships to one another. Such a model could be useful in understanding complex disturbances of pain perception.

\section{Acknowledgments}

We are grateful to Shannon Gallagher, Edward McLaurin, Caitlin Murray, and Eric W. Owings for their help in carrying out this study. Vanessa Miller, Dr. Pei Feng Lim, and Dr. Muhammad Q. Siddiqi recruited and screened 
the participants. Thanks are also due to Mike Young and Ollie Monbureau for expert technical support. This research was supported by NINDS grant NS045685.

\section{References}

1. Andrew D, Greenspan JD. Peripheral coding of tonic mechanical cutaneous pain: Comparison of nociceptor activity in rat and human psychophysics. J Neurophysiol. 1999; 82:2641-8. [PubMed: 10561433]

2. Coghill RC, McHaffie JG, Yen Y-F. Neural correlates of interindividual differences in the subjective experience of pain. Proc Natl Acad Sci Unit States Am. 2003; 100:8538-42.

3. Dallenbach KM. Pain: history and present status. Amer J Psychol. 1939; 52:331-47.

4. Dworkin SF, LeResche L. Research diagnostic criteria for temporomandibular disorders: Review, criteria, examinations and specifications, critique. J Craniomandib Disord: Facial \& Oral Pain. 1992; 6:301-55.

5. Gescheider, GA.; Wright, JH.; Verrillo, RT. Information-processing channels in the tactile sensory system. New York (NY): Psychology Press; 2009.

6. Greene LC, Hardy JD. Adaptation of thermal pain in the skin. J Appl Physiol. 1962; 17:693-6. [PubMed: 13901526]

7. Greffrath W, Baumgärtner U, Treede R-D. Peripheral and central components of habituation of heat pain perception and evoked potentials in humans. Pain. 2007; 132:301-11. [PubMed: 17533117]

8. Hollins M, Harper D, Gallagher S, Owings EW, Lim PF, Miller V, Siddiqi MQ, Maixner W. Perceived intensity and unpleasantness of cutaneous and auditory stimuli: An evaluation of the generalized hypervigilance hypothesis. Pain. 2009; 141:215-21. [PubMed: 19121558]

9. Hollins M, Roy EA, Crane SA. Vibratory antinociception: Effects of vibration amplitude and frequency. J Pain. 2003; 4:381-91. [PubMed: 14622680]

10. Hollins M, Sigurdsson A. Vibrotactile amplitude and frequency discrimination in temporomandibular disorders. Pain. 1998; 75:59-67. [PubMed: 9539674]

11. Hollins M, Sigurdsson A, Fillingim L, Goble A. Vibrotactile threshold is elevated in temporomandibular disorders. Pain. 1996; 67:89-96. [PubMed: 8895235]

12. King CD, Wong F, Currie T, Mauderli AP, Fillingim RB, Riley JL 3rd. Deficiency in endogenous modulation of prolonged heat pain in patients with irritable bowel syndrome and temporomandibular disorder. Pain. 2009; 143:172-8. [PubMed: 19278784]

13. Kosek, E. Disturbances of pain perception in fibromyalgia. In: Lautenbacher, S.; Fillingim, RB., editors. Pathophysiology of pain perception. New York (NY): Kluwer Academic/Plenum Publishers; 2004. p. 77-91.

14. Kosek E, Hansson P. Modulatory influence on somatosensory perception from vibration and heterotopic noxious conditioning stimulation (HNCS) in fibromyalgia patients and healthy subjects. Pain. 1997; 70:41-51. [PubMed: 9106808]

15. LaMotte RH, Torebjörk HE, Robinson CJ, Thalhammer JG. Time-intensity profiles of cutaneous pain in normal and hyperalgesic skin: A comparison with C-fiber nociceptive activities in monkey and human. J Neurophysiol. 1984; 51:1434-50. [PubMed: 6737035]

16. Lundeberg T, Nordemar R, Ottoson D. Pain alleviation by vibratory stimulation. Pain. 1984; 20:25-44. [PubMed: 6333660]

17. Maixner W, Fillingim R, Sigurdsson A, Kincaid S, Silva S. Sensitivity of patients with painful temporomandibular disorders to experimentally evoked pain: evidence for altered temporal summation of pain. Pain. 1998; 76:71-81. [PubMed: 9696460]

18. Melzack R. The short-form McGill Pain Questionnaire. Pain. 1987; 30:191-8. [PubMed: 3670870]

19. Melzack R, Wall PD. Pain mechanisms: A new theory. Science. 1965; 150:971-9. [PubMed: 5320816]

20. Mendell LM, Wall PD. Responses of single dorsal cord cells to peripheral cutaneous unmyelinated fibers. Nature. 1965; 206:97-9. [PubMed: 14334366]

21. Naert ALG, Kehlet H, Kupers R. Characterization of a novel model of tonic heat pain stimulation in healthy volunteers. Pain. 2008; 138:163-71. [PubMed: 18207325]

22. Pennebaker, JW. The psychology of physical symptoms. New York (NY): Springer- Verlag; 1982. 
23. Pertovaara A. Modification of human pain threshold by specific tactile receptors. Acta Physiol Scand. 1979; 107:339-41. [PubMed: 232366]

24. Price DD. Characteristics of second pain and flexion reflexes indicative of prolonged central summation. Exp Neurol. 1972; 37:371-87. [PubMed: 4637957]

25. Price DD, Hu JW, Dubner R, Gracely RH. Peripheral suppression of first pain and central summation of second pain evoked by noxious heat pulses. Pain. 1977; 3:57-68. [PubMed: 876667]

26. Price DD, Staud R, Robinson ME, Mauderli AP, Cannon R, Vierck CJ. Enhanced temporal summation of second pain and its central modulation in fibromyalgia patients. Pain. 2002; 99:4959. [PubMed: 12237183]

27. Rhudy JL, France CR, Bartley EJ, Williams AE, McCabe KM, Russell JL. Does pain catastrophizing moderate the relationship between spinal nociceptive processes and pain sensitivity? J Pain. 2009; 10:860-9. [PubMed: 19638330]

28. Roy EA, Hollins M, Maixner W. Reduction of TMD pain by high-frequency vibration: a spatial and temporal analysis. Pain. 2003; 101:267-74. [PubMed: 12583869]

29. Salter MW, Henry JL. Evidence that adenosine mediates the depression of spinal dorsal horn neurons induced by peripheral vibration in the cat. Neuroscience. 1987; 22:631-50. [PubMed: 3670602]

30. Staud R, Vierck CJ, Cannon RL, Mauderli AP, Price DD. Abnormal sensitization and temporal summation of second pain (wind-up) in patients with fibromyalgia syndrome. Pain. 2001; 91:16575. [PubMed: 11240089]

31. Sullivan MJL, Thibault P, Andrikonyte J, Butler H, Catchlove R, Larivière C. Psychological influences on repetition-induced summation of activity-related pain in patients with chronic low back pain. Pain. 2009; 141:70-8. [PubMed: 19059719]

32. Treede R, Meyer RA, Campbell JN. Myelinated mechanically insensitive afferents from monkey hairy skin: Heat-response properties. J Neurophysiol. 1998; 80:1082-93. [PubMed: 9744923]

33. Vierck CJ Jr. Mechanisms underlying development of spatially distributed chronic pain (fibromyalgia). Pain. 2006; 124:242-63. [PubMed: 16842915]

34. Wall PD, Cronly-Dillon JR. Pain, itch, and vibration. Arch Neurol. 1960; 2:365-75. [PubMed: 13842563]

35. Ward L, Wright E, McMahon SB. A comparison of the effects of noxious and innocuous counterstimuli on experimentally induced itch and pain. Pain. 1996; 64:129-38. [PubMed: 8867255]

36. Wolfe F, Smythe HA, Yunus MB, Bennett RM, Bombardier C, Goldenberg DL, Tugwell P, Campbell SM, Abeles M, Clark P, Fam AG, Farber SJ, Fiechtner JJ, Franklin CM, Gatter RA, Hamaty D, Lessard J, Lichtbroun AS, Masi AT, McCain GA, Reynolds WJ, Romano TJ, Russell IJ, Sheoin RP. The American College of Rheumatology 1990 criteria for the classification of fibromyalgia. Report of the Multicenter Criteria Committee. Arthritis Rheum. 1990; 33:160-72. [PubMed: 2306288]

37. Yarnitsky D, Kunin M, Brik R, Sprecher E. Vibration reduces thermal pain in adjacent dermatomes. Pain. 1997; 69:75-7. [PubMed: 9060015] 


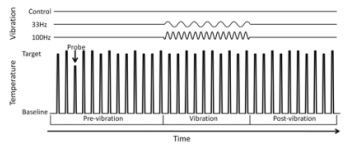

\section{Figure 1.}

Timeline of an experimental run. Each run consisted of 33 heat pulses, each $3 \mathrm{~s}$ in duration and separated by $12 \mathrm{~s}$ at a baseline temperature of $38^{\circ} \mathrm{C}$. The subject rated the pain intensity of each pulse verbally using a 0-100 scale. Two temperatures varying by $0.5^{\circ} \mathrm{C}$ (selected for each subject individually on the basis of previous measurements) were pseudo-randomly interspersed, except for the third (probe) trial, in which a temperature $2{ }^{\circ} \mathrm{C}$ lower than the low target temperature was used. The probe trial is not included in subsequent figures. Each subject participated in three runs: one with $33 \mathrm{~Hz}$ vibration, one with $100 \mathrm{~Hz}$ vibration, and one without vibration. The order of these runs was counterbalanced within each group. During runs with vibration present, the vibration started immediately after the subject rated the $12^{\text {th }}$ pulse of heat (not including the probe trial), and continued at a constant amplitude until the subject verbally reported the pain of the $22^{\text {nd }}$ pulse. 


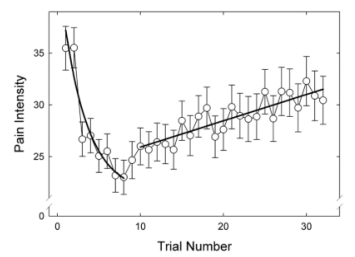

Figure 2.

Time course of changes in responsiveness within a control (i.e., no-vibration) run consisting of 32 trials spaced at 15-s intervals. Each point is the pain intensity rating for one trial, averaged across all participants; error bars show \pm 1 S.E.M. Stimulus temperature was adjusted for each subject to initially produce moderate pain. An initial drop in responsiveness was followed by a second phase during which pain increased for most subjects. The exponential curve drawn through the data of the first 8 trials represents the adaptation process. A regression line, representing slow temporal summation, has been fit to the data for trials $10-32$. The two temperatures (differing by $0.5^{\circ} \mathrm{C}$ ) employed for a given participant were pseudo-randomly ordered across trials, except that the lower of the two was always used on trial 1, and the higher on trial 2. 


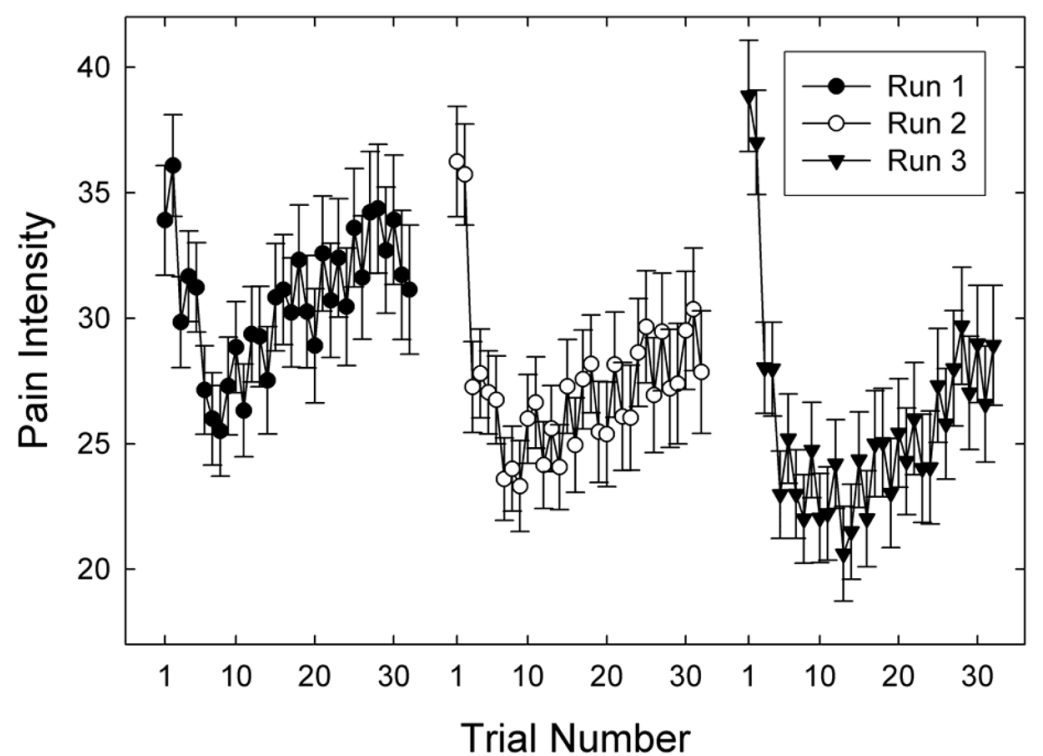

Fig. 3.

Pain ratings for the three runs constituting the experimental session, averaged across all participants (FM, TMD, and HC). Breaks of 10 min separated consecutive runs. The order of the three types of runs $(33 \mathrm{~Hz}, 100 \mathrm{~Hz}$, and control) varied randomly across participants, subject to the constraint that each of the six possible orders occurred about equally often for members of each of the three clinical groups. The data shown here for each run (e.g. Run 1) are therefore averaged across runs of different types. Error bars represent \pm 1 S.E.M. 


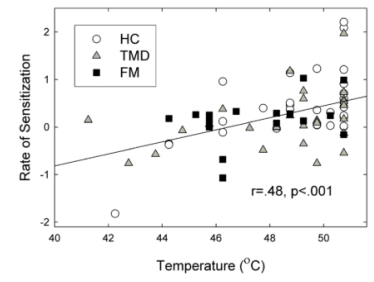

Fig. 4.

The rate of sensitization is plotted as a function of stimulus temperature. Rate is expressed in units of change (on the 0-100 pain intensity scale) per trial. Different symbols show the results for subjects with FM (squares), TMD (triangles), and for healthy controls (circles). Temperature for each subject is the mean of the two values used intermittently. Since regression slopes for the three groups were similar, a regression line fit to all the data is shown. 


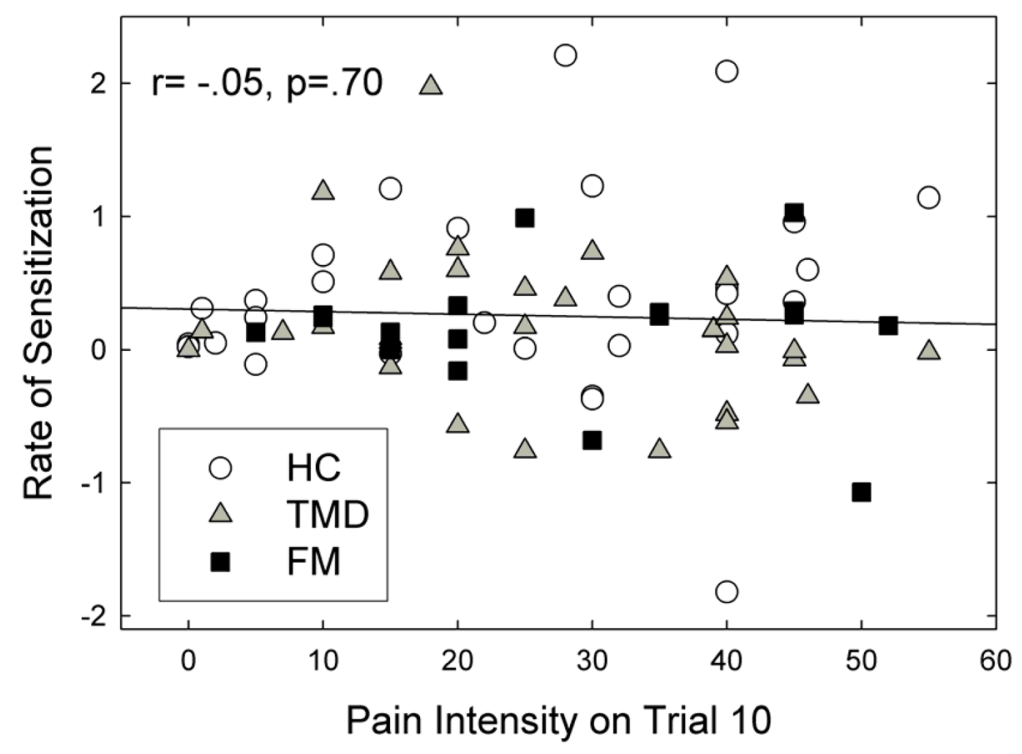

Fig. 5.

The rate of sensitization is plotted as a function of pain intensity rating on the first trial of the series over which this rate was calculated. Different symbols show the results for subjects with FM (squares), TMD (triangles), and for healthy controls (circles). 


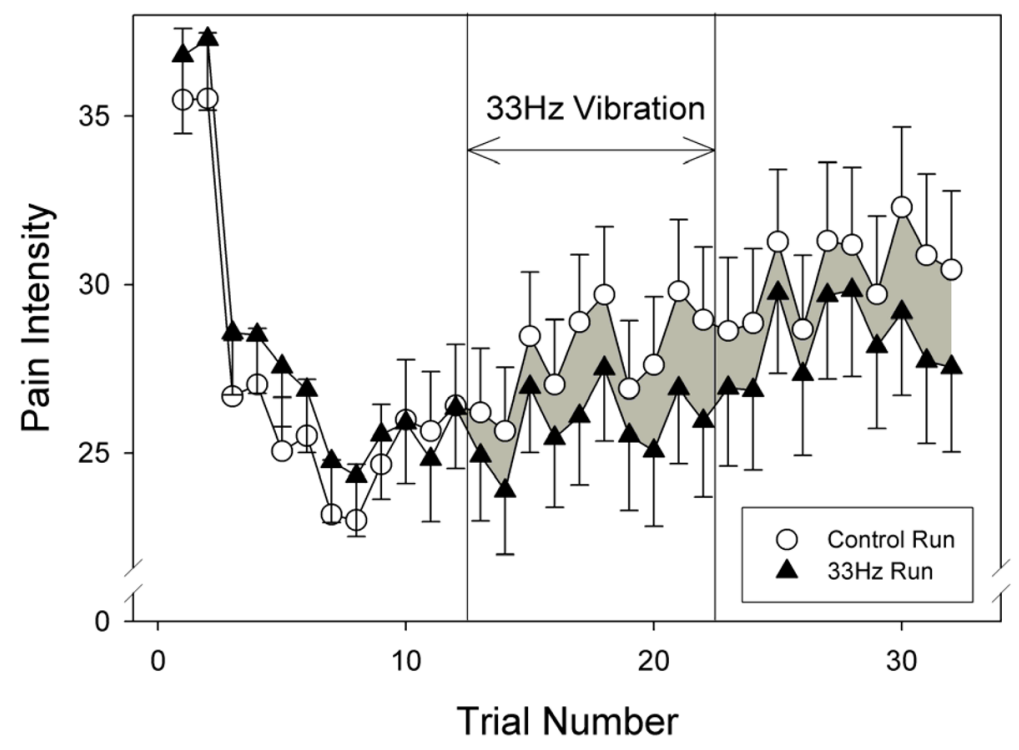

Fig. 6.

Pain intensity ratings over the course of control runs (circles), and runs in which $33 \mathrm{~Hz}$ vibration was present during trials 13-22 (triangles). Data are averaged across all participants (FM, TMD, and HC). The order of the two types of runs was counterbalanced within each group. The area between the functions during and after the vibration period is shaded, to show the extent of vibratory pain modulation. Error bars represent \pm 1 S.E.M. 